\title{
Numerical analysis of transport properties of boron-doped graphene FETs
}

\author{
Paolo Marconcini*, Gianluca Fiori*, Alessandro Ferretti ${ }^{\dagger}$, Giuseppe Iannaccone*, and Massimo Macucci* \\ * Dipartimento di Ingegneria dell'Informazione, \\ Università di Pisa, \\ Via Caruso 16, I-56122, Pisa, Italy \\ Email: macucci@mercurio.iet.unipi.it \\ $\dagger$ IPCF-CNR, \\ Via Moruzzi 1, I-56124, Pisa, Italy
}

\begin{abstract}
We have performed a numerical investigation of the effect of boron doping on the dispersion relations of armchair graphene nanoribbons, finding that it should reduce the strong variability of the energy gap predicted for atomic-scale fluctuations of the nanoribbon width. We also present the transport characteristics that we have obtained, within a self-consistent Non Equilibrium Green's Function (NEGF) simulation, for a fieldeffect transistor based on boron-doped graphene nanoribbons. As a consequence of doping, mobility, and thus the current through the device, are suppressed, but there seems to be a possibility to mitigate this adverse effect, by locating the dopants near the edges of the nanoribbon, their energetically favored position.
\end{abstract}

\section{INTRODUCTION}

Graphene represents a very promising material for electronic applications, because of its high carrier mobility, of ballistic transport over rather long distances, and of its planar nature, which is suitable for fabrication with lithographic processes analogous to those for traditional microelectronics.

However, it is well known that a graphene sheet has a zero energy gap, which prevents the direct fabrication of field effect devices useful for digital circuit applications, because it is not possible to achieve a significant $I_{\text {on }} / I_{\text {off }}$ ratio. A proposed approach for the creation of a bandgap consists in the lithographic definition of a narrow stripe of graphene, the so-called graphene nanoribbon, which has been shown to exhibit a nonzero energy gap, thereby making it possible to shut down the channel by means of the electrostatic action of the gate. Although the bandgap induced by lateral confinement may represent a solution, acceptable transistor behavior is expected only for very small widths (below $5 \mathrm{~nm}$ ) that are very difficult to achieve with standard lithographic techniques; in addition, the thus achieved bandgap has a strong dependence on the exact number of atoms in the nanoribbon cross-section. Indeed, in the absence of edge distortion, an armchair nanoribbon characterized by $N$ dimer lines across its width would be metallic if $N=3 M-1$, with $M$ an integer number, while otherwise a nonzero energy gap would exist [1]. If the effect of edge distortion (i.e. the reduction of the interatomic distance along the nanoribbon edges) is taken into account [2], [3], an energy gap appears for all the values of $N$, but its amplitude strongly depends on the value of $\bmod (N, 3)$, in particular assuming a minimum value when
$N=3 M-1$ (quasi-metallic nanoribbon). On the average, the value of the bandgap decreases when the width of the nanoribbon increases, in such a way as to approach the zero limit value of an infinite sheet of graphene.

It is apparent that, even if a technology to reliably define very narrow nanoribbons were available, it would not be possible to control the width with atomic precision, and therefore severe variability in the characteristics would be expected. A preliminary investigation that we have performed indicates that doping the nanoribbons acts upon the bandgap and the device characteristics in such a way that the variability associated with the width of the ribbon modulo 3 is significantly reduced. Substitutional doping with boron atoms is considered, and is shown to dramatically improve the bandgap of quasi-metallic nanoribbons, while having a moderate effect on semiconducting ones (as long as narrow nanoribbons, with a width below $10 \mathrm{~nm}$, are considered). The drawback is represented by a drop in mobility due to scattering against the dopants. Our results show that this negative effect nearly disappears if the dopants are moved close to the edges of the nanoribbon. As long as the total dopant concentration is kept unchanged, relocation of the dopants in the boundary region does not reduce significantly the bandgap with respect to uniform doping.

In this preliminary investigation periodic boron patterning has been taken into consideration, which, although practically very hard to implement, makes the calculation of an actual bandgap possible, for purposes of direct comparison with the undoped case. In a real-world situation doping will most likely be random (although possibly located only along the boundaries) and therefore the actual device behavior will be a statistical average of the possible arrangements of dopant atoms.

\section{TIGHT-BINDING ANALYSIS}

We use a simple nearest-neighbor tight-binding model and transport is computed by means of the Non Equilibrium Green's function approach. The first step consisted in determining the values of the tight-binding parameters that best describe, within a nearest-neighbor model, the Hamiltonian of a graphene nanoribbon doped with boron atoms, in substitutional positions. 


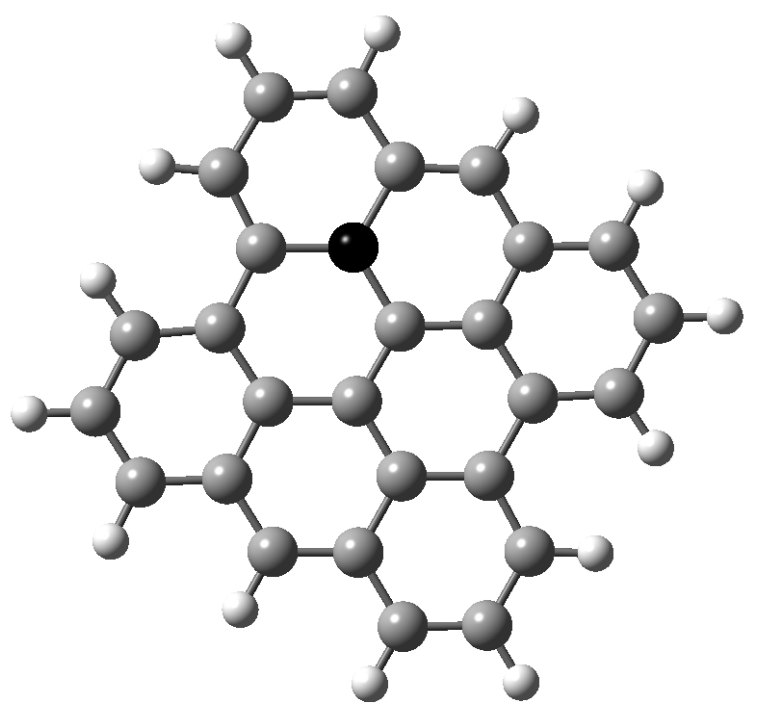

Fig. 1. Cluster of 27 carbon atoms (in grey) with a boron dopant atom (in black), passivated with 14 hydrogen atoms (in white).

The value of the parameters has been determined by fitting the molecular orbital eigenvalues from a tight-binding calculation on hydrogen-passivated clusters of various sizes with those obtained for the same clusters with a Density Functional Theory (DFT) calculation. Progressively larger doped graphene clusters have been considered, with different positions of the dopants. As an example, a cluster with a single boron atom in an internal position is shown in Fig. 1. The DFT work has been performed with the software Gaussian03 [4], using the $6-311 \mathrm{G}^{*}$ basis set of gaussian-type orbitals and the B3LYP functional. The geometry of each cluster has been optimized, minimizing the total energy of the structure, with an unrestricted calculation. The discrete energy levels of the obtained structure have then been computed using a restricted open shell formalism, in order to force neglect of the differences between the two spin components of the wave functions and thus to achieve a simplified spin-independent description. Out of the overall set of energy levels, only the $\pi$ molecular orbitals, mainly deriving from the $p_{z}$ atomic orbitals and characterized by a nodal plane in correspondence of the graphene plane, have been considered, since they are delocalized and give the main contribution to electrical conduction. We have then fitted, with the Hook and Jeeves algorithm [5], the energy levels associated with the completely occupied molecular orbitals found from the DFT analysis of the considered clusters with those obtained from a tightbinding study of the same structures. In our tight-binding model we have considered only interactions between nearestneighbor atoms, including however the effect of the variation of the bond length at the edges of the nanoribbon, which has been shown to have a considerable effect on the band structure, opening up an energy gap also in otherwise metallic ribbons [2], [3]. In particular, due to the similarities between
TABLE I

THE VALUES OF THE TIGHT-BINDING PARAMETERS OBTAINED FROM THE FITTING PROCEDURE.

\begin{tabular}{|c||c|}
\hline TB parameter & value in $\mathrm{eV}$ \\
\hline$\varepsilon_{C}$ & 0.0 \\
$\varepsilon_{B}$ & 2.1574 \\
$t_{C C}$ & -2.8838 \\
$t_{C C_{\text {edge }}}$ & -3.0225 \\
$t_{C B}$ & -2.0232 \\
$t_{C B_{\text {edge }}}$ & -2.1205 \\
\hline
\end{tabular}

the carbon and boron atoms, we have assumed an identical percentage variation of the transfer integral at the edges of the structure between carbon atoms and between carbon and boron atoms. In Tab. I we report the values of the tight-binding parameters obtained from our fitting procedure. Contrary to what has happened for the carbon parameters, we have noticed some dependence of the obtained boron parameters on the position of the dopant; we have therefore considered average parameters, assuming that in an actual device the parameters from all the different boron positions will contribute to the final transport results.

We have then used these values to perform a nearestneighbor tight-binding analysis of an infinitely long armchair graphene nanoribbon, hydrogen passivated and periodically doped with boron atoms. In Fig. 2 we show the energy dispersion relations of graphene nanoribbons with $N=14$, 15 , and 16 with (solid line) and without (dashed line) boron doping $(7.14 \%$ for $N=14,6.66 \%$ for $N=15$, and $6.25 \%$ for $N=16$ ). In the figures the wave vectors are normalized with respect to $1 /\left(3 a_{C C}\right)$, where $a_{C C}$ is the distance between nearest-neighbor carbon atoms inside the nanoribbon.

In these simulations we have assumed to dope and periodically repeat the unit cell of the original undoped graphene nanoribbon, but the results do not undergo big changes if the dopants are distributed on larger unit cells. As can be clearly seen, boron doping definitely increases the bandgap if the number $N$ of dimer lines across the width of the nanoribbon can be expressed as $3 M-1$, i.e., for the nanoribbons with the smallest energy gap in undoped conditions, while it produces a small increase or even a reduction of the bandgap in the other cases. This clearly decreases the modulo 3 dependence of the bandgap on the exact value of $N$, thus making the electronic properties of the nanoribbons more robust with respect to imperfection in the fabrication process.

As we will discuss in the following section, introduction of dopants in the bulk of the nanoribbon has an adverse effect on the transport properties, due to the additional scattering; we have then analyzed the effect on the bandgap of concentrating all the dopant atoms along the edges of the nanoribbon. Results are presented in Fig. 3, for the same overall concentrations of boron atoms as for the data reported in Fig. 2: for the particular choice of positions for the boron atoms, the effect on the bandgap is even increased for the quasi-metallic nanoribbon, and in general the behavior is similar to that observed for bulk 

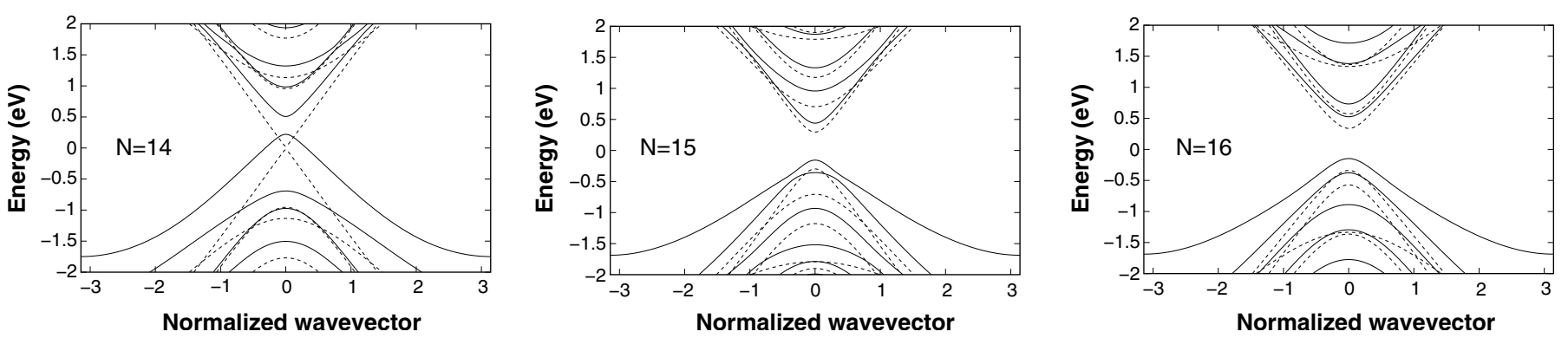

Fig. 2. Dispersion relations of graphene nanoribbons with $N=14,15$ and 16 , with a boron doping concentration equal to $7.14 \%$ (for $N=14$ ), $6.66 \%$ (for $N=15$ ) and $6.25 \%$ (for $N=16$ ).
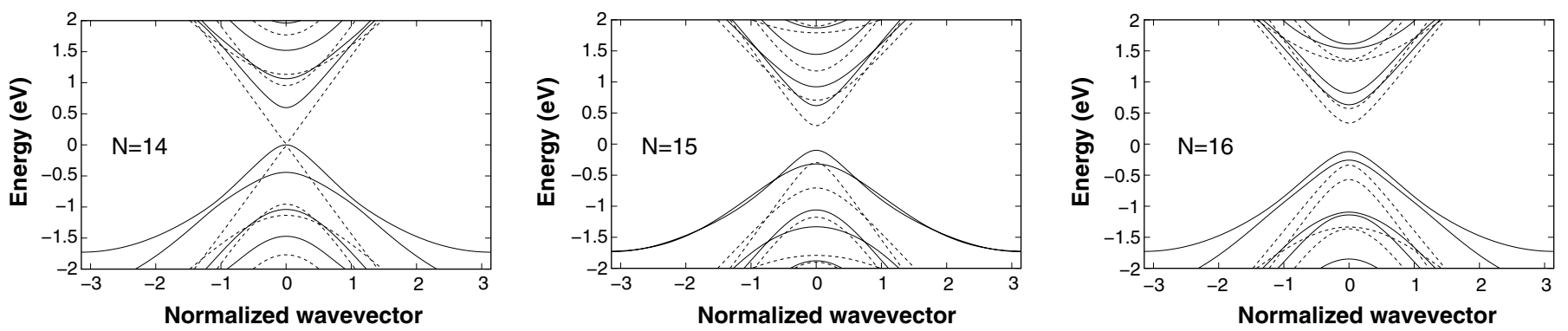

Fig. 3. Dispersion relations of graphene nanoribbons with $N=14,15$ and 16, with the same doping concentration as in Fig. 2. In this case however the dopants are located on the boundaries of the ribbon.

doping.

From unrestricted calculations on boron doped clusters, we have noticed that the clusters with the boron atom in an edge position are always the most stable. For example, for the case of Fig. 1, the clusters with the boron atom in a position different from the edge are characterized by an increase in energy (up to $10 \mathrm{Kcal} / \mathrm{mol}$ ). Therefore carbon nanoribbons with boron atoms near the edges should be energetically favored, in agreement with previous calculations [6].

\section{STUDY OF TRANSPORT IN FETS BASED ON BORON-DOPED GRAPHENE NANORIBBONS}

We have then investigated the transport properties of fieldeffect transistors based on boron-doped graphene nanoribbons, exploiting the capabilities of our in-house developed code NanoTCAD ViDES [7]. In particular, the open-boundary Schrödinger equation is solved self-consistently with the threedimensional Poisson equation, within the Non Equilibrium Green's function (NEGF) formalism. The Hamiltonian is expressed on a $p_{z}$ atomic orbital basis set in the real space, using the fitted tight-binding parameters, and in particular modifying the hopping parameters and the on-site energies in correspondence of substitutional doping atoms according to Tab. I. Schottky contacts with a Schottky barrier equal to $E_{\text {gap }} / 2$ (with $E_{\text {gap }}$ the ribbon energy gap) are considered at GNR ends (as presently happens in most of the experimental devices), introducing appropriate self-energies as in [8]. Transport is assumed to be completely ballistic. From a numerical point of view, the nonlinear system is solved by means of the Newton-Raphson method, coupled with the Gummel iterative scheme [9].

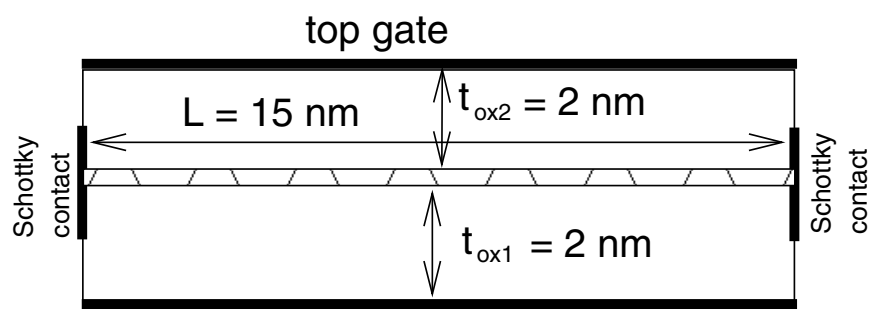

bottom gate

Fig. 4. Structure of the simulated double-gate graphene nanoribbon FET: the channel length is $15 \mathrm{~nm}$, the oxide thickness is $2 \mathrm{~nm}$ and Schottky contacts are considered at GNR ends.

In detail, we have considered a transistor with a metallic double gate and with a $2 \mathrm{~nm}$ thick oxide (Fig. 4). The channel consists of an $(N-1) a_{C C}(\sqrt{3} / 2)$-wide armchair graphene nanoribbon and is $15 \mathrm{~nm}$ long. In our simulations we have considered two different values of $N: N=14$ (that is of the type $3 M-1$ ) and $N=16$, corresponding to a quasi-metallic and to a semiconducting nanoribbon, respectively. A concentration of boron dopants around 5\% has been considered.

In Fig. 5 we show the output characteristics of the transistor for $N=16$. In this case, the device already has an acceptable behavior without doping; with bulk doping of $5 \%$ the ON current decreases by a factor of 3 (in other cases of bulk doping we do observe a much stronger suppression of transport), while for edge doping the full ON current is recovered and even an improvement in the $I_{\mathrm{ON}} / I_{\mathrm{OFF}}$ ratio is observed. The overall concentration of dopant atoms is kept about the same for bulk and edge doping. 


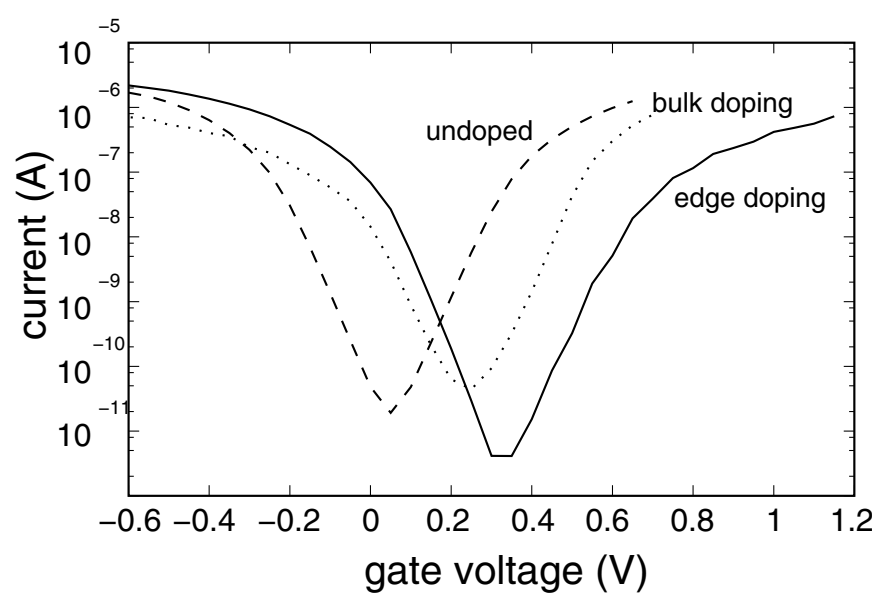

Fig. 5. Drain current of a graphene nanoribbon FET with $N=16$, for $V_{D S}=0.1 \mathrm{~V}$, as a function of the gate voltage. Curves are presented for an undoped nanoribbon, bulk doping (5\%) and edge doping (5\%).

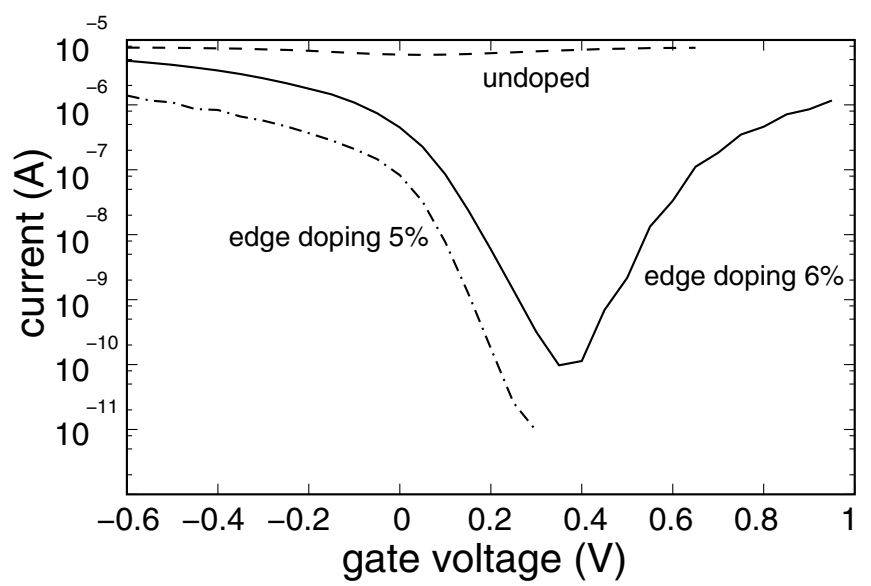

Fig. 6. Drain current of a graphene nanoribbon FET with $N=14$, for $V_{D S}=0.1 \mathrm{~V}$, as a function of the gate voltage. Curves are presented for an undoped nanoribbon, and two different schemes of edge doping (5\% and $6 \%)$.

In Fig. 6 we report the output characteristics for a nanoribbon FET with $N=14$ (quasi-metallic case): in this case the behavior for the undoped case is unacceptable, with almost no modulation of the current, while with edge doping (presented for two arrangements of the boron atoms along the edges, with a concentration of $5 \%$ and $6 \%$ ) a good transistor behavior is observed. The curve for a doping concentration of $5 \%$ is reported only up to $V_{G S} \approx 0.3 \mathrm{~V}$, because of convergence problems beyond that point.

\section{CONCLUSIONS}

Our preliminary results indicate that it should be possible to decrease the dependence on small variations of the ribbon width of the transport properties of a graphene nanoribbon field-effect transistor by doping it with boron atoms. In particular, improved performance seems to be achievable with edge doping. An improvement of the $I_{O N} / I_{O F F}$ ratio is observed, in particular for the case of nanoribbons with $N=3 M-1$ with $M$ integer, which have a small energy gap in the absence of doping. The presence of boron atoms only close to the nanoribbon boundaries prevents suppression of the ON current, which is otherwise the consequence of the high doping levels required to obtain a significant effect on the bandgap. However extensive work is still needed, both in order to improve the fitting procedure used to determine the tight-binding parameters, possibly switching to a thirdneighbor or even more sophisticated approach, and in order to find ways to further increase control of the channel, up to the values required for proper operation of digital circuits. It is anyway clear that doping cannot be a substitute for advanced lithographic techniques capable of defining nanoribbons with widths of the order of $5 \mathrm{~nm}$ or less, since lateral confinement will still represent the main source of bandgap opening, while doping can help in easing the precision requirements and the problems associated with edge roughness.

\section{ACKNOWLEDGEMENT}

Support from the EC Project GRAND (Contract No. 215752) is gratefully acknowledged.

\section{REFERENCES}

[1] K. Nakada, M. Fujita, G. Dresselhaus, and M. Dresselhaus, "Edge states in graphene ribbons: Nanometer size effect and edge shape dependence," Phys. Rev. Lett., vol. 54, pp. 17954-17961, 1996.

[2] M. Fujita, M. Igami, and K. Nakada, "Lattice distortion in nanographite ribbons,” J. Phys. Soc. Jpn., vol. 66, pp. 1864-1867, 1997.

[3] Y.-W. Son, M. L. Cohen, and S. G. Louie, "Energy Gaps in Graphene Nanoribbons," Phys. Rev. Lett., vol. 97, p. 216803, 2006.

[4] Gaussian 03, M. J. Frisch et al., 2003.

[5] R. Hooke and T. A. Jeeves, "“Direct Search" Solution of Numerical and Statistical Problems," J. Assoc. Comp. Mach., vol. 8, pp. 212-229, 1961.

[6] T. B. Martins, R. H. Miwa, A. J. R. da Silva, and A. Fazzio, "Electronic and Transport Properties of Boron-Doped Graphene Nanoribbons," Phys. Rev. Lett., vol. 98, p. 196803, 2007.

[7] NanoTCAD ViDES is available online at: www.nanohub.org/tools/vides

[8] Y. Yoon, G. Fiori, S. Hong, J. Guo, and G. Iannaccone, "Performance Comparison of Graphene Nanoribbon FETs With Schottky Contacts and Doped Reservoirs," IEEE Trans. Electron Devices, vol. 55, pp. 2314-2323, 2008.

[9] G. Fiori, G. Iannaccone, and G. Klimeck, "A three-dimensional simulation study of the performance of carbon nanotube field-effect transistors with doped reservoirs and realistic geometry," IEEE Trans. Electron Devices, vol. 53, pp. $1782-1788,2006$ 